OPEN ACCESS

Edited by:

Michael Piper,

The University of Queensland,

Australia

Reviewed by: Tomasz Nowakowski,

University of California, San

Francisco, United States

Laura Fenlon,

The University of Queensland,

Australia

*Correspondence:

Lei Xing

xing@mpi-cbg.de

Wieland B. Huttner

huttner@mpi-cbg.de

Specialty section:

This article was submitted to

Stem Cell Research,

a section of the journal

Frontiers in Cell and Developmental

Biology

Received: 01 April 2020

Accepted: 29 April 2020

Published: 26 May 2020

Citation:

Xing L and Huttner WB (2020)

Neurotransmitters as Modulators of Neural Progenitor Cell Proliferation

During Mammalian Neocortex

Development.

Front. Cell Dev. Biol. 8:391.

doi: 10.3389/fcell.2020.00391

\section{Neurotransmitters as Modulators of Neural Progenitor Cell Proliferation During Mammalian Neocortex Development}

\author{
Lei Xing* and Wieland B. Huttner* \\ Max Planck Institute of Molecular Cell Biology and Genetics, Dresden, Germany
}

Neural progenitor cells (NPCs) play a central role during the development and evolution of the mammalian neocortex. Precise temporal and spatial control of NPC proliferation by a concert of cell-intrinsic and cell-extrinsic factors is essential for the correct formation and proper function of the neocortex. In this review, we focus on the regulation of NPC proliferation by neurotransmitters, which act as a group of cell-extrinsic factors during mammalian neocortex development. We first summarize, from both in vivo and in vitro studies, our current knowledge on how $\gamma$-aminobutyric acid (GABA), glutamate and serotonin modulate NPC proliferation in the developing neocortex and the potential involvements of different receptors in the underlying mechanisms. Another focus of this review is to discuss future perspectives using conditionally gene-modified mice and human brain organoids as model systems to further our understanding on the contribution of neurotransmitters to the development of a normal neocortex, as well as how dysregulated neurotransmitter signaling leads to developmental and psychiatric disorders.

Keywords: neocortex, neurotransmitter, neural progenitor cell, cell proliferation, development

\section{INTRODUCTION}

During mammalian brain development, the formation of the central nervous system (CNS) results from a series of events, which begins with the neural induction and the proliferation of the NPCs (Goodman and Shatz, 1993). In the early developing neocortex, neuroepithelial cells (NECs) function as the primary NPCs and undergo symmetric proliferative divisions to expand the neocortical NPC pool (Götz and Huttner, 2005; Lui et al., 2011; Florio and Huttner, 2014). At the onset of neurogenesis, NECs transform into apical (or ventricular) radial glia (aRG), which undergo mitosis at the ventricular surface and reside in the ventricular zone (VZ) of the developing neocortex (Götz and Huttner, 2005; Rakic, 2009; Lui et al., 2011; Florio and Huttner, 2014; WilschBräuninger et al., 2016). In virtually all mammals, aRG are thought to possess high proliferative capacity to both amplify themselves and give rise to basal progenitors (BPs), including basal intermediate progenitors (bIPs) and basal (or outer) radial glia (bRG) (Götz and Huttner, 2005; Rakic, 2009; Fietz et al., 2010; Hansen et al., 2010; Lui et al., 2011; Reillo et al., 2011; Florio and Huttner, 2014; Wilsch-Bräuninger et al., 2016). BPs delaminate from the ventricular surface and migrate to the subventricular zone (SVZ), where they typically reside and undergo mitosis to give rise to cortical neurons, which are destined for six different cortical layers (Götz and Huttner, 2005; 
Rakic, 2009; Lui et al., 2011; Florio and Huttner, 2014; WilschBräuninger et al., 2016). At later stages of development, either following neurogenesis or concomitant with still ongoing neuron production, NPCs switch their fate to generate glial cells, such as astrocytes and oligodendrocytes (Lee et al., 2000). In order to guarantee the proper construction of the complex neocortex, each step in this developmental sequence must be under precise spatial and temporal regulation. While significant progress has been made in understanding how NPC-intrinsic factors contribute to a balanced NPC proliferation, there are still open questions about the regulation of NPC proliferation by environmental cues, such as neurotransmitters.

Among several categories of cell-extrinsic signals, neurotransmitters have gained attention as important factors to influence CNS development (Cameron et al., 1998; Nguyen et al., 2001; Ojeda and Avila, 2019), although the classic role of neurotransmitters is in neuronal communication by acting as synaptic chemical messengers in the mature CNS. Indeed, neurotransmitters mediate developmental processes such as cell proliferation (Haydar et al., 2000), neuronal differentiation (Salazar et al., 2008), neuronal migration (Komuro and Rakic, 1993; Murthy et al., 2014), synaptic maturation (Fu et al., 2012), neurite growth (Anelli et al., 2013) and cell death (Ikonomidou et al., 2001). For example, serotonin controls the migration of caudal ganglionic eminence-derived interneurons into the neocortex (Murthy et al., 2014). The GABA receptors along developing inhibitory axons sense GABA release and promote presynaptic maturation to shape the pattern of synapse formation and distribution (Fu et al., 2012). Glutamate induces neuronal apoptosis, which is mediated via activation of calpain and caspase- 3 proteases as well as the translocation of apoptosis inducing factor (Zhang and Bhavnani, 2006). Several recent studies strongly suggest that neurotransmitters could act as growth regulators or morphogen-like signaling molecules to regulate NPC proliferation during cortical development (Represa and Ben-Ari, 2005; Côté et al., 2007). In this review, we summarize our current knowledge on the regulation of neocortical NPC proliferation by different neurotransmitters during mammalian brain development and discuss future research perspectives in studying the involvement of neurotransmitters in neocortical development under both physiological and pathological conditions. We do not discuss in detail the synthesis and metabolism of any individual neurotransmitter, nor their role in other developmental processes beside progenitor proliferation, as these aspects have been intensively reviewed previously (Cameron et al., 1998; Nguyen et al., 2001; Represa and Ben-Ari, 2005; Ojeda and Avila, 2019).

\section{NPC PROLIFERATION REGULATED BY NEUROTRANSMITTERS}

\section{GABA}

During mammalian brain development, GABA, the main inhibitory neurotransmitter in the mature brain, excites cortical cells due to the high expression level of the $\mathrm{Na}^{+}-\mathrm{K}^{+}-2 \mathrm{Cl}^{-}$ cotransporter (NKCC1) (Hübner et al., 2001) and low expression level of $\mathrm{K}^{+}-\mathrm{Cl}^{-}$transporter member five (KCC2) (Owens and Kriegstein, 2002; Lee et al., 2005). As one of the most abundant neurotransmitters detected in the developing brain, GABA appears in the germinal zones, intermediate zone and layer I of the cortical plate during early stages of development (Haydar et al., 2000). Starting as early as E9.5 in mice, the GABAergic neurons generated from subcortical structures are gradually migrating into the developing neocortex, and these neurons could serve as the source of releasable GABA in the neocortical wall (Tanaka and Nakajima, 2012).

Although GABA is the most studied neurotransmitter in the context of regulating the proliferation of NPCs, there is apparent controversy about the trophic effect of GABA during neocortical development. Upon binding of GABA to $\mathrm{GABA}_{A}$ receptors, which in cultured E16-E19 rat neocortical tissue explants have been shown to be expressed in the VZ NPCs (presumably in aRG), $\mathrm{Cl}^{-}$ions diffuse through these ion channels along their concentration gradient (LoTurco et al., 1995). The NPCs in the VZ of developing rat neocortex thus lose intracellular $\mathrm{Cl}^{-}$, which leads to membrane depolarization and the increase of intracellular $\mathrm{Ca}^{2+}$ concentration through the activation of voltage-gated calcium channels (VGCCs) (LoTurco et al., 1995). This increase of intracellular $\mathrm{Ca}^{2+}$ concentration, induced by GABA, is potentially involved in the inhibition of DNA synthesis of VZ NPCs and decreases their proliferation rate in the cultured tissue explants of developing rat neocortex (LoTurco et al., 1995). The same study also reported that the effects of GABA in inhibiting DNA synthesis in VZ NPCs can be blocked by modulating the $\mathrm{Cl}^{-}$concentration using a $\mathrm{GABA}_{A}$ receptor antagonist (LoTurco et al., 1995). In line with this, another study (Andang et al., 2008) suggested that GABA inhibits cell cycle progression and therefore decreases proliferation of mouse embryonic stem cells and neural crest stem cells, which express glutamic acid decarboxylase (GAD) and functional $\mathrm{GABA}_{A}$ receptors. The underlying mechanisms include phosphorylation of the critical factor in the S/G2 DNA-damage checkpoint complex, histone $\mathrm{H} 2 \mathrm{AX}$, by phosphatidylinositol-3-OH-kinase-related kinase (PIKK) upon membrane hyperpolarization following $\mathrm{GABA}_{A}$ receptor activation (Andang et al., 2008). It has recently been shown that mouse VZ NPCs become more hyperpolarized at later developmental stages and that experimental membrane hyperpolarization shifts the transcriptional program and division mode of VZ NPCs to a later developmental stage, in which VZ NPCs generate two daughter IPs instead of amplifying themselves (Vitali et al., 2018).

However, it has also been reported that $\mathrm{GABA}_{A}$ receptor activation stimulates cell proliferation and renewal in a culture system of isolated NPCs from developing mouse brain. The increased proliferation rate was found to be due to an upregulation of ciliary neurotrophic factor (CNTF) receptor expression, which in turn enhanced the trophic effect of CNTF (Fukui et al., 2008b). A follow-up study from the same research group further showed that $\mathrm{GABA}_{B}$ receptor activation led to a significant increase in the capacity of isolated mouse cortical NPCs in forming neurospheres, which has been supported by 
the analyses of $\mathrm{GABA}_{B}$ R1-null mice (Fukui et al., 2008a). Thus, in the isolated mouse NPC culture system, GABA seems to be able to increase the proliferation of these progenitors through two separate mechanisms involving the recruitment of different types of GABA receptors and different growth-stimulating factors. The synthesis and release of growth factors and neuropeptides from NPCs in the developing neocortex can indeed be stimulated by neurotransmitters and may play a role in regulating NPC proliferation together with neurotransmitters (Fukui et al., 2008b; Yuzwa et al., 2016).

Regarding the contradicting findings between these studies, obvious explanations beside species differences would be the micro-environmental difference between tissue explant culture and isolated NPC culture, where different NPC populations are being studied, as well as the difference in developmental stage. Nevertheless, all these in vitro studies suggest that there is a direct effect of GABA in regulating NPC proliferation, with the direction of the effect being species-, region- and environmentdependent. However, surprisingly, gene-modified mice which have only $0.02 \%$ of GABA circulating in the embryonic brain due to the knockdown of the GABA-synthesizing enzymes GAD65 and GAD67 did not show altered brain histogenesis, including cortical layering (Ji et al., 1999). A possible explanation of the lack of adverse phenotypes could be that other neurotransmitter systems compensate for the malfunction induced by the loss of GABA, including modulation of cortical NPC proliferation and migration, possibly by glutamate and glycine, both of which are able to depolarize NPCs in the germinal zones of developing rodent neocortex (LoTurco et al., 1995; Flint et al., 1998). In addition, a more rigorous evaluation of cellular morphology and ultrastructure, cell density as well as the cellular composition of the developing neocortex is needed to further uncover developmental defects of these GAD-knockdown mice.

The alterations in proliferation of neocortical NPCs induced by the external application of GABA in vitro demonstrated that GABA has the potential to directly regulate NPC proliferation, a conclusion consistent with the finding that the opposite effects are observed upon blocking GABA receptors (LoTurco et al., 1995). This suggests that endogenously synthesized GABA in the developing neocortex regulates neurogenesis in rodent germinal zones, including both VZ and SVZ. Interestingly, the effects of GABA on NPC proliferation are completely opposite in the VZ NPCs (aRG) versus SVZ NPCs (BPs) of developing mouse neocortex, potentially due to activation of different receptor subtypes (Figure 1) and triggering different signaling mechanisms (Haydar et al., 2000). For example, the most highly expressed GABA receptor subunits in the mouse NPC populations are GABRA2 and GABRG2, both of which showed a relatively higher expression level in BPs (bRG and bIP) compared to APs (aRG). Thus, depending on the in vitro experimental conditions or the in vivo environment that the NPCs reside in, GABA signaling may exhibit different impacts on the proliferation of NPCs in developing neocortex.

\section{Glutamate}

Glutamate is the main excitatory neurotransmitter in the mature CNS. Through binding to different types of receptors, glutamate is essential for maintaining various cognitive functions including learning and memory (Riedel et al., 2003; Mattson, 2008). Glutamate receptors can be categorized into two main classes: (1) ionotropic glutamate receptors (iGluR), which include three types of receptors: N-methyl-D-aspartate (NMDA) receptors, $\alpha$ amino-3-hydroxy-5-methylisoxazole-4-propionic acid (AMPA) receptors, and kainic acid (KA) receptors; and (2) metabotropic glutamate receptors 1-8 (mGluR1-8) (Riedel et al., 2003).

During development, glutamate is detectable in the germinal zones of developing mouse neocortex as early as E12, potentially released by the Cajal-Retzius cells in the marginal zone (del Rio et al., 1995; Haydar et al., 2000). Among iGluRs, AMPA/KA receptors are the first ones to appear and are highly expressed by NPCs in the germinal zones of the embryonic rodent and fetal human neocortex (Figure 1) (LoTurco et al., 1995; Haydar et al., 2000; Maric et al., 2000). Through activating AMPA/KA receptors, glutamate decreases DNA synthesis of the NPCs in the germinal zones, and hence their proliferation, in rat organotypic slice cultures (LoTurco et al., 1995; Haydar et al., 2000).

The NMDA receptor is also involved in regulating NPC proliferation in developing mouse neocortex, albeit indirectly. Calcium imaging in cultured mouse neocortical slices suggested that MAP2-positive cortical neurons, but not nestin-positive NPCs in the VZ, are responsive to an NMDA antagonist (Hirasawa et al., 2003). Through regulating the expression levels of components of the Notch pathway and increasing the synthesis of brain-derived neurotrophic factor (BDNF), chronic exposure to the NMDA antagonist caused sustained proliferation of NPCs in the VZ (Hirasawa et al., 2003). In line with the finding that NMDA receptor activation inhibits cortical NPC proliferation in the developing mouse neocortex, NPCs isolated from developing rat neocortex, which are believed to transiently express NMDA receptor subunits, also showed a decreased proliferation when exposed to an NMDA receptor agonist (Yoneyama et al., 2008).

In contrast, elongated GFAP-positive NPCs, presumably radial glial cells, that express NMDA receptor subunits, dissociated from fetal human neocortex, responded to glutamate and an NMDA antagonist in a completely opposite manner compared to mouse NPCs. Glutamate significantly enhanced the proliferation rate of isolated human NPCs in vitro, and the increased proliferation could be inhibited by a specific NMDA receptor antagonist (Suzuki et al., 2006). The same study also showed that AMPA receptors, KA receptors and mGluRs are most likely not involved in the proliferation of radial glial cells induced by glutamate (Suzuki et al., 2006).

Among the mGluRs, it has been reported that mGluR5 is involved in the modulation of NPC proliferation in developing rat and human neocortex, where this receptor is expressed (Figure 1) (Boer et al., 2010; Zhao et al., 2011, 2012). In human, mGluR5 activation stimulates both ERK and JNK pathways, which leads to promotion of NPC proliferation. The human NPCs with activated mGluR5 also showed an increased level of cyclin D1, which results in cell cycle progression underlying the increased proliferation of NPCs (Zhao et al., 2011). In mouse, blocking mGluR5 function by a selective mGluR5 antagonist reduced proliferation and increased cell death of mouse forebrain NPCs during development, while the 


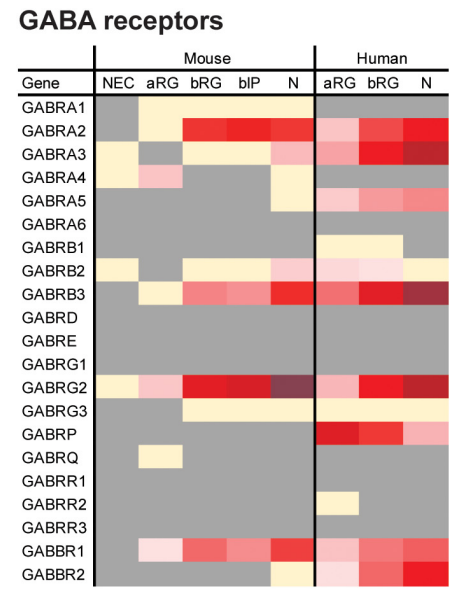

\section{Serotonin receptors}

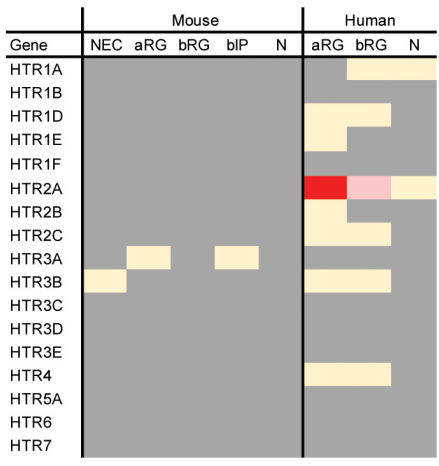

\section{Cannabinoid receptors}

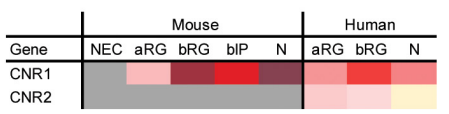

Glutamate receptors

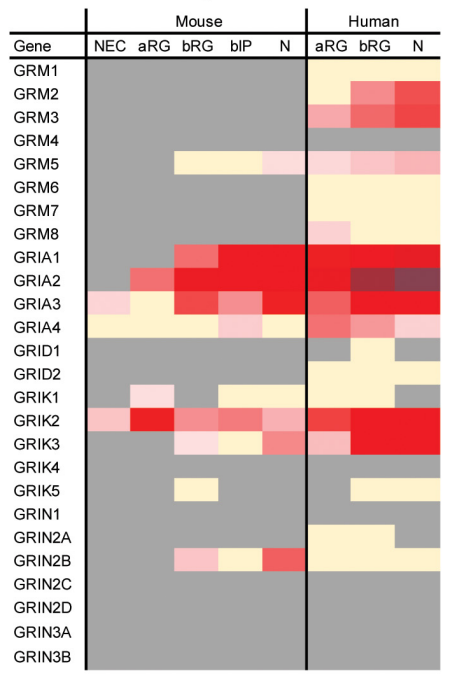

Adrenergic receptors

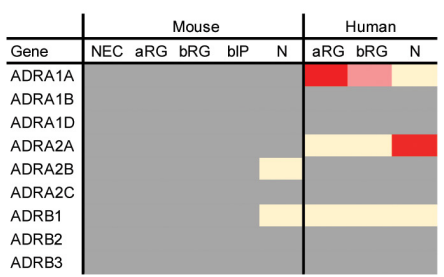

\section{Glycine receptors}

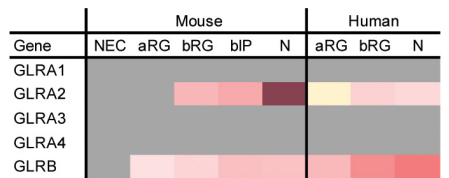

Cholinergic receptors

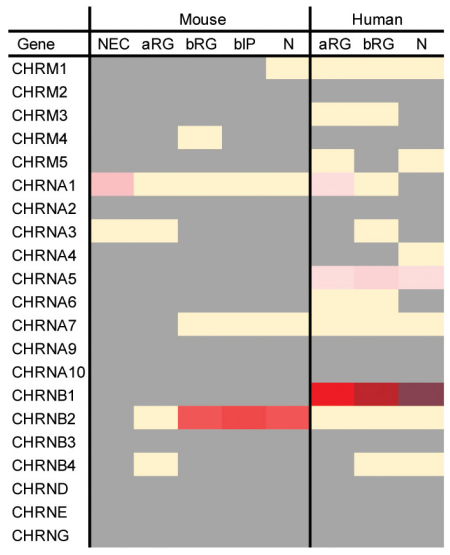

Purinergic receptors

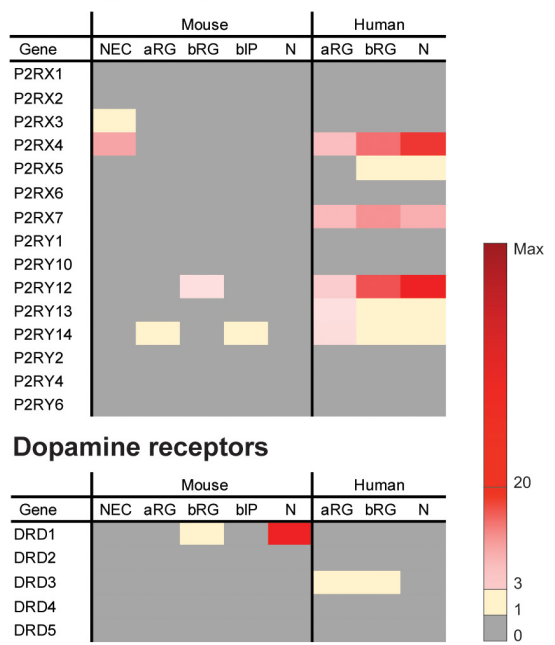

FIGURE 1 | Previously published sets of transcriptomic data (Florio et al., 2015; Albert et al., 2017) were analyzed here for the mRNA expression levels of neurotransmitter receptors in embryonic mouse and fetal human neocortex. FPKM values of neurotransmitter receptors in the indicated isolated cell populations of embryonic mouse (E9.5 for NEC, E14.5 for aRG, bRG, bIP and N) and fetal human (12-13 wpc) neocortex are indicated by the color scale shown at bottom right. Note that $\mathrm{N}$ (neuronal fraction) in fetal human neocortex includes bRG in the G1 phase of the cell cycle. Key observations from the analyses can be summarized as follows. (1) Four neurotransmitter receptors, GRIA3, GRIK2, CHRNA1, and P2RX4, were found to be expressed in mouse NECs, however, at low levels (FPKM $=3.9$, $5.1,6.6,5.5$, respectively). This suggests that the involvement of neurotransmitter signaling in NEC expansion during mouse cortical development is presumably limited. (2) Of the neurotransmitter receptors that are expressed in both embryonic mouse and fetal human neocortex, the majority showed the highest expression levels in the N fraction, such as GABRA2, GABRB3, GABRG2, GRIA2, GRIK3, and GLRA2. These receptors are most likely expressed on the cell surface of neurons where they may receive the respective neurotransmitter signal. (3) All human neurotransmitter receptor-encoding genes presented in the figure have orthologs in mouse. Thus, an expression observed in one species but not the other indicates a differential expression pattern of the neurotransmitter receptor between cortical cells in mouse and those in human. For example, GABRA5, GABBR2, GRM2, GRM3, CHRNB1, and ADRA2A are potentially involved in neuronal functions only in human, but not mouse, during neocortical development. In contrast, GRIN2B, CHRNB2, and DRD1 are potentially involved in neuronal functions only in mouse, but not human, during neocortical development. (4) Of the neurotransmitter receptors only expressed in fetal human but not embryonic mouse neocortex, GABRP, HTR2A, ADRA1A, P2RX7, and CNR2 showed a greater expression in $\mathrm{RRG}$ and/or bRG than in N, which raises the possibility that the activation of these receptors could be of relevance for NPC proliferation during the development and even the evolutionary expansion of the human neocortex.

activation of mGluR5 increased the number of proliferating NPCs (Di Giorgi-Gerevini et al., 2005). In line with this, NPCs of mGluR5 knockout mice also exhibited decreased proliferation compared to those of wildtype mice, both in vitro and in vivo (Di Giorgi-Gerevini et al., 2005).

\section{Serotonin}

Serotonin has been postulated to exert a role in cortical development, as cortical serotonin arises from placental sources at the onset of neurogenesis and from embryonic serotonergic afferents at later developmental stages in both mouse and human (Bonnin et al., 2011). Even though both the endogenous serotonin system in the embryonic hindbrain and placenta can be sources to supply the embryonic forebrain with sufficient serotonin starting from E10.5 and throughout the development of the mouse brain (Bonnin et al., 2011), there are no serotonin receptors, of any subtype, expressed at significant levels in the germinal zones of the developing mouse neocortex (Figure 1) 
to receive and amplify the readily available serotonin signals (Bonnin et al., 2011; Fietz et al., 2012; Florio et al., 2015). This has also been suggested by data from early in vitro studies using a rat organotypic slice culture system, which have shown no effect of serotonin on cortical NPC proliferation as the number of BrdU-labeled cells were similar between serotonin-treated and untreated rat neocortex slices (Dooley et al., 1997).

However, in vivo studies aiming to understand the effects of serotonin on cortical development using transgenic mouse models with altered serotonin levels in the embryonic neocortex have suggested that the proliferation rate of cortical progenitors is decreased by serotonin (Côté et al., 2007; Cheng et al., 2010). A double knockout mouse model for the serotonin-degrading enzymes, monoamine oxidase A (MAOA) and monoamine oxidase B (MAOB), exhibited significant reductions in Sox2positive cells and Tbr2-positive bIPs in the SVZ at E17.5 and P2, but not at earlier developmental stages (Cheng et al., 2010). Although MAO metabolizes both serotonin and dopamine, it was suggested that the decrease in NPC abundance in MAO knockout mice was indeed caused by the increased level of serotonin, not dopamine (Cheng et al., 2010). In contrast, however, a knockout mouse model for the serotonin-synthesizing enzyme tryptophan hydroxylase 1 (TPH1) also showed a decreased number of BrdUpositive cortical progenitors in the VZ (Côté et al., 2007), which leaves the role of serotonin in NPC proliferation unclear.

Compared to the contribution of serotonin and its receptors to neuronal migration and maturation, for which there are several studies, very little is known about the effects of serotonin on the proliferation of cortical NPCs. Recent comparative transcriptomic studies have revealed a differential expression pattern of serotonin receptor 2A (HTR2A) in cortical NPCs between mouse and human (Florio et al., 2015; Mayer et al., 2019), which may point to a potential role of serotonin and HTR2A in regulating proliferation of human NPCs. However, no effects on progenitor proliferation have been observed when treating cultured human neocortical slices with one particular specific HTR2A agonist (Mayer et al., 2019). More thorough studies using other agonists or serotonin are needed before reaching a final conclusion, especially due to the fact that multiple pathways are coupled to HTR2A receptor activation. In line with this, a recent study (Farrelly et al., 2019) identified a direct role of serotonin, which was independent from its function in neurotransmission and cellular signaling, in modifying histone proteins and, consequently, regulating gene expression. Findings on histone serotonylation have revealed a wide array of mechanisms for future investigations on cortical NPC proliferation modulated by serotonin (Farrelly et al., 2019).

\section{OUTLOOK AND FUTURE RESEARCH DIRECTIONS}

Over the past few years, our view of NPCs during neocortical development has massively changed. The advancements in neuroimaging and single-cell transcriptomic analyses have enabled us to reveal more detailed profiling and characterization of different NPC types in different mammalian species (Fietz et al., 2012; Pollen et al., 2014;
Florio et al., 2015; Nowakowski et al., 2017). This has provided foundations for further studies on the regulation of proliferation of different NPC types by neurotransmitters, and potentially in different model systems. This is true, in particular, when there are differential expression patterns of neurotransmitter receptors among different NPC populations or among different species, such as between mouse and human (Figure 1).

\section{Conditionally Gene-Modified Mouse Models}

Various genetically engineered mouse models with disrupted neurotransmitter signaling have been generated to study the role of neurotransmitters in brain development (Ji et al., 1999; Di Giorgi-Gerevini et al., 2005; Côté et al., 2007; Cheng et al., 2010). However, systematically knocking out neurotransmittersynthesizing or -degrading enzymes and neurotransmitter receptors in the whole organism is not ideal for studying the developing neocortex, since depleting or elevating the level of a particular neurotransmitter could potentially induce secondary alterations that may also bear significant impact on cortical development. Thus, it might be necessary to generate conditionally gene-modified mouse models that allow disruption of neurotransmitter signaling in a temporally and spatially more controlled manner. For example, glutamate decreases NPC proliferation through AMPA receptor activation, but increases NPC proliferation through mGluR5 activation (LoTurco et al., 1995; Di Giorgi-Gerevini et al., 2005). By conditionally knocking out the respective AMPA receptor and overexpressing mGluR5 exclusively in one specific NPC type, the proliferation-inhibiting AMPA receptor-coupled signaling could be abolished and the mGluR5-induced proliferation-stimulating signaling could be amplified, with the level of glutamate in the gene-modified animal remaining the same. These conditionally gene-modified mouse models could provide us with much more insight into the molecular mechanism of glutamate-regulated NPC proliferation and allow us to focus on studying the effects of glutamate in one particular NPC type.

\section{Human Brain Organoids as a Model System}

Compared to human, rodents such as mouse and rat, the most commonly used experimental mammalian animals in developmental neuroscience, have a relatively small and smooth (lissencephalic) neocortex. In contrast, many primates, including human, have a folded (gyrencephalic) neocortex that is expanded in size (Florio and Huttner, 2014). Furthermore, the proportion of bRG among the BPs and their proliferative capacity are dramatically different between rodent and human (Fietz et al., 2010; Hansen et al., 2010). Therefore, in order to understand how human neocortex grows during development and expands during evolution, it is necessary to study the influence of neurotransmitters on NPC proliferation in fetal human neocortex. The development of brain organoids (Kadoshima et al., 2013; Lancaster et al., 2013) has opened up new avenues to study human neocortex development and evolution as well as neurodevelopmental disorders. Human brain organoids serve 
as a good, although not ideal, model which mimics certain aspects of the cytoarchitecture and cell-type composition of the developing human neocortex. Potential applications of wildtype and gene-modified brain organoids are feasible for studying the roles of neurotransmitters and their receptors in human NPC proliferation ex vivo.

\section{Neurotransmitters, Neocortex Malformations and Psychiatric Disorders}

Neocortex malformations, which are thought to be caused by alterations of NPC proliferation and abundance, are featured in several neurological or psychiatric disorders such as epilepsy, Down syndrome and autism spectrum disorders (ASD) (Pinson et al., 2019). Some of these disorders also show altered levels of neurotransmitters in the CNS. For example, autism patients show a deceased level of GABA in the left perisylvian region of the auditory cortex (Rojas et al., 2014), and GABA receptor subunit genes on chromosome 15q11-q13 are considered risk factors for autistic disorders (Ashley-Koch et al., 2006). Moreover, altered levels of glutamate and serotonin as well as the functional deficiency or dysregulation of their receptors have also been suspected to be involved in ASD (Jamain et al., 2002; Yang et al., 2014; Zheng et al., 2016). It is plausible that the above mentioned neurotransmitter imbalance in ASD is caused by neocortex malformations with reduced abundance of neurotransmitter-secreting neurons, especially in cases linking maternal drug intake or exposure to developmental toxicants to neurodevelopmental disorders. For example, prenatal exposure to ethanol causes a spectrum of physical and mental dysfunctions in children, including pre- and postnatal growth delay, microcephaly, mental retardation and various behavioral abnormalities, which are due to the loss of specific cortical neurons and dysregulation of neuronal migration, such as GABAergic neurons (Shenoda, 2017), inhibition of the neurotrophic properties of glutamate, or the activation of specific GABA receptors (Ikonomidou et al., 2000).

One interesting question that can be raised from connecting neocortex malformations, induced by dysregulated NPC proliferation, with altered levels of neurotransmitters in

\section{REFERENCES}

Albert, M., Kalebic, N., Florio, M., Lakshmanaperumal, N., Haffner, C., Brandl, H., et al. (2017). Epigenome profiling and editing of neocortical progenitor cells during development. EMBO J. 36, 2642-2658. doi: 10.15252/embj.201796764

Andang, M., Hjerling-Leffler, J., Moliner, A., Lundgren, T. K., Castelo-Branco, G., Nanou, E., et al. (2008). Histone H2AX-dependent GABA(A) receptor regulation of stem cell proliferation. Nature 451, 460-464. doi: 10.1038/ nature 06488

Anelli, T., Cardarelli, S., Ori, M., Nardi, I., Biagioni, S., and Poiana, G. (2013). 5-Hydroxytryptamine $1 \mathrm{~A}$ and $2 \mathrm{~B}$ serotonin receptors in neurite outgrowth: involvement of early growth response protein 1. Dev. Neurosci. 35, 450-460. doi: $10.1159 / 000354423$

Ashley-Koch, A. E., Mei, H., Jaworski, J., Ma, D. Q., Ritchie, M. D., Menold, M. M., et al. (2006). An analysis paradigm for investigating multi-locus effects in complex disease: examination of three GABA receptor subunit genes on developmental disorders is: Could disrupted neurotransmitter signaling during cortical development be the causative factor for disorders like autism? To further understand the influences of neurotransmitters in neurodevelopmental disorders like autism, we can now take advantage of the option to generate cerebral organoids from patient-derived induced pluripotent stem cells (iPSCs) to model the disorder and study the neocortex malformation and neurotransmitter imbalance involved in the disorder.

\section{CONCLUDING REMARKS}

Over the past few years, the dissection of NPC cell biology during the development of the mammalian neocortex has given us substantial insights into the spatial and temporal control mechanisms of NPC proliferation by the concert of cell-intrinsic and cell-extrinsic factors. Studies on the developmental actions of neurotransmitters have also further advanced our understanding on how the growth of the neocortex can be affected by these extrinsic factors. Looking forward, with promising concepts and platforms being established, more comprehensive and integrative interpretations on how neurotransmitters maintain normal CNS development and protect against cortical dysfunction could be achieved. Learning more about the roles that neurotransmitters play during human cortical development will not only provide valuable knowledge for understanding our own cognitive abilities, but also shed light on the development of pharmacological interventions against a number of human neurodevelopmental disorders.

\section{AUTHOR CONTRIBUTIONS}

LX and WH wrote and edited the manuscript.

\section{FUNDING}

WH was supported by grants from the DFG (SFB 655, A2), the ERC (250197) and ERA-NET NEURON (MicroKin).

15q11-q13 as risk factors for autistic disorder. Ann. Hum. Genet. 70, 281-292. doi: 10.1111/j.1469-1809.2006.00253.x

Boer, K., Encha-Razavi, F., Sinico, M., and Aronica, E. (2010). Differential distribution of group I metabotropic glutamate receptors in developing human cortex. Brain Res. 1324, 24-33. doi: 10.1016/j.brainres.2010. 02.005

Bonnin, A., Goeden, N., Chen, K., Wilson, M. L., King, J., Shih, J. C., et al. (2011). A transient placental source of serotonin for the fetal forebrain. Nature 472, 347-350. doi: 10.1038/nature09972

Cameron, H. A., Hazel, T. G., and Mckay, R. D. (1998). Regulation of neurogenesis by growth factors and neurotransmitters. J. Neurobiol. 36, 287-306.

Cheng, A., Scott, A. L., Ladenheim, B., Chen, K., Ouyang, X., Lathia, J. D., et al. (2010). Monoamine oxidases regulate telencephalic neural progenitors in late embryonic and early postnatal development. J. Neurosci. 30, 10752-10762. doi: 10.1523/JNEUROSCI.2037-10.2010 
Côté, F., Fligny, C., Bayard, E., Launay, J. M., Gershon, M. D., Mallet, J., et al. (2007). Maternal serotonin is crucial for murine embryonic development. Proc. Natl. Acad. Sci. U.S.A. 104, 329-334. doi: 10.1073/pnas.0606722104

del Rio, J. A., Martinez, A., Fonseca, M., Auladell, C., and Soriano, E. (1995). Glutamate-like immunoreactivity and fate of Cajal-Retzius cells in the murine cortex as identified with calretinin antibody. Cereb. Cortex 5, 13-21. doi: $10.1093 /$ cercor/5.1.13

Di Giorgi-Gerevini, V., Melchiorri, D., Battaglia, G., Ricci-Vitiani, L., Ciceroni, C., Busceti, C. L., et al. (2005). Endogenous activation of metabotropic glutamate receptors supports the proliferation and survival of neural progenitor cells. Cell Death Differ. 12, 1124-1133. doi: 10.1038/sj.cdd.4401639

Dooley, A. E., Pappas, I. S., and Parnavelas, J. G. (1997). Serotonin promotes the survival of cortical glutamatergic neurons in vitro. Exp. Neurol. 148, 205-214. doi: 10.1006/exnr.1997.6633

Farrelly, L. A., Thompson, R. E., Zhao, S., Lepack, A. E., Lyu, Y., Bhanu, N. V., et al. (2019). Histone serotonylation is a permissive modification that enhances TFIID binding to H3K4me3. Nature 567, 535-539. doi: 10.1038/s41586-0191024-7

Fietz, S. A., Kelava, I., Vogt, J., Wilsch-Brauninger, M., Stenzel, D., Fish, J. L., et al. (2010). OSVZ progenitors of human and ferret neocortex are epithelial-like and expand by integrin signaling. Nat. Neurosci. 13, 690-699. doi: 10.1038/nn.2553

Fietz, S. A., Lachmann, R., Brandl, H., Kircher, M., Samusik, N., Schroder, R., et al. (2012). Transcriptomes of germinal zones of human and mouse fetal neocortex suggest a role of extracellular matrix in progenitor self-renewal. Proc. Natl. Acad. Sci. U.S.A. 109, 11836-11841. doi: 10.1073/pnas.1209647109

Flint, A. C., Liu, X., and Kriegstein, A. R. (1998). Nonsynaptic glycine receptor activation during early neocortical development. Neuron 20, 43-53. doi: 10 . 1016/s0896-6273(00)80433-x

Florio, M., Albert, M., Taverna, E., Namba, T., Brandl, H., Lewitus, E., et al. (2015). Human-specific gene ARHGAP11B promotes basal progenitor amplification and neocortex expansion. Science 347, 1465-1470. doi: 10.1126/science. aaa 1975

Florio, M., and Huttner, W. B. (2014). Neural progenitors, neurogenesis and the evolution of the neocortex. Development 141, 2182-2194. doi: 10.1242/dev. 090571

Fu, Y., Wu, X., Lu, J., and Huang, Z. J. (2012). Presynaptic GABA(B) receptor regulates activity-dependent maturation and patterning of inhibitory synapses through dynamic allocation of synaptic vesicles. Front. Cell Neurosci. 6:57. doi: 10.3389/fncel.2012.00057

Fukui, M., Nakamichi, N., Yoneyama, M., Ozawa, S., Fujimori, S., Takahata, Y., et al. (2008a). Modulation of cellular proliferation and differentiation through $\mathrm{GABA}(\mathrm{B})$ receptors expressed by undifferentiated neural progenitor cells isolated from fetal mouse brain. J. Cell. Physiol. 216, 507-519. doi: 10.1002/ jcp. 21422

Fukui, M., Nakamichi, N., Yoneyama, M., Yoshida, K., Ozawa, S., Kitayama, T., et al. (2008b). Up-regulation of ciliary neurotrophic factor receptor expression by GABAA receptors in undifferentiated neural progenitors of fetal mouse brain. J. Neurosci. Res. 86, 2615-2623. doi: 10.1002/jnr.21708

Goodman, C. S., and Shatz, C. J. (1993). Developmental mechanisms that generate precise patterns of neuronal connectivity. Cell 72(Suppl.), 77-98. doi: 10.1016/ s0092-8674(05)80030-3

Götz, M., and Huttner, W. B. (2005). The cell biology of neurogenesis. Nat. Rev. Mol. Cell Biol. 6, 777-788.

Hansen, D. V., Lui, J. H., Parker, P. R., and Kriegstein, A. R. (2010). Neurogenic radial glia in the outer subventricular zone of human neocortex. Nature 464, 554-561. doi: 10.1038/nature08845

Haydar, T. F., Wang, F., Schwartz, M. L., and Rakic, P. (2000). Differential modulation of proliferation in the neocortical ventricular and subventricular zones. J. Neurosci. 20, 5764-5774. doi: 10.1523/JNEUROSCI.20-15-05764.2000

Hirasawa, T., Wada, H., Kohsaka, S., and Uchino, S. (2003). Inhibition of NMDA receptors induces delayed neuronal maturation and sustained proliferation of progenitor cells during neocortical development. J. Neurosci. Res. 74, 676-687. doi: $10.1002 /$ jnr. 10795

Hübner, C. A., Lorke, D. E., and Hermans-Borgmeyer, I. (2001). Expression of the Na-K-2Cl-cotransporter NKCC1 during mouse development. Mech. Dev. 102, 267-269. doi: 10.1016/s0925-4773(01)00309-4

Ikonomidou, C., Bittigau, P., Ishimaru, M. J., Wozniak, D. F., Koch, C., Genz, K., et al. (2000). Ethanol-induced apoptotic neurodegeneration and fetal alcohol syndrome. Science 287, 1056-1060. doi: 10.1126/science.287.5455. 1056

Ikonomidou, C., Bittigau, P., Koch, C., Genz, K., Hoerster, F., Felderhoff-Mueser, U., et al. (2001). Neurotransmitters and apoptosis in the developing brain. Biochem. Pharmacol. 62, 401-405.

Jamain, S., Betancur, C., Quach, H., Philippe, A., Fellous, M., Giros, B., et al. (2002). Linkage and association of the glutamate receptor 6 gene with autism. Mol. Psychiatry 7, 302-310. doi: 10.1038/sj.mp.4000979

Ji, F., Kanbara, N., and Obata, K. (1999). GABA and histogenesis in fetal and neonatal mouse brain lacking both the isoforms of glutamic acid decarboxylase. Neurosci. Res. 33, 187-194. doi: 10.1016/s0168-0102(99)00011-5

Kadoshima, T., Sakaguchi, H., Nakano, T., Soen, M., Ando, S., Eiraku, M., et al. (2013). Self-organization of axial polarity, inside-out layer pattern, and speciesspecific progenitor dynamics in human ES cell-derived neocortex. Proc. Natl. Acad. Sci. U.S.A. 110, 20284-20289. doi: 10.1073/pnas.1315710110

Komuro, H., and Rakic, P. (1993). Modulation of neuronal migration by NMDA receptors. Science 260, 95-97. doi: 10.1126/science.8096653

Lancaster, M. A., Renner, M., Martin, C. A., Wenzel, D., Bicknell, L. S., Hurles, M. E., et al. (2013). Cerebral organoids model human brain development and microcephaly. Nature 501, 373-379. doi: 10.1038/nature12517

Lee, H., Chen, C. X., Liu, Y. J., Aizenman, E., and Kandler, K. (2005). KCC2 expression in immature rat cortical neurons is sufficient to switch the polarity of GABA responses. Eur. J. Neurosci. 21, 2593-2599. doi: 10.1111/j.1460-9568. 2005.04084.x

Lee, J. C., Mayer-Proschel, M., and Rao, M. S. (2000). Gliogenesis in the central nervous system. Glia 30, 105-121.

LoTurco, J., Owens, D., Heat, M., Davis, M., and Kriegstein, A. (1995). GABA and glutamate depolarize cortical progenitor cells and inhibit DNA synthesis. Neuron 15, 1287-1298. doi: 10.1016/0896-6273(95)90008-x

Lui, J. H., Hansen, D. V., and Kriegstein, A. R. (2011). Development and evolution of the human neocortex. Cell 146, 18-36. doi: 10.1016/j.cell.2011.06.030

Maric, D., Liu, Q. Y., Grant, G. M., Andreadis, J. D., Hu, Q., Chang, Y. H., et al. (2000). Functional ionotropic glutamate receptors emerge during terminal cell division and early neuronal differentiation of rat neuroepithelial cells. J. Neurosci. Res. 61, 652-662. doi: 10.1002/1097-4547(20000915)61:6\&lt;652:: AID-JNR9\&gt;3.0.CO;2-J

Mattson, M. P. (2008). Glutamate and neurotrophic factors in neuronal plasticity and disease. Ann. N. Y. Acad. Sci. 1144, 97-112. doi: 10.1196/annals.14 18.005

Mayer, S., Chen, J., Velmeshev, D., Mayer, A., Eze, U. C., Bhaduri, A., et al. (2019). Multimodal single-cell analysis reveals physiological maturation in the developing human neocortex. Neuron 102, 143-158. doi: 10.1016/j.neuron. 2019.01.027

Murthy, S., Niquille, M., Hurni, N., Limoni, G., Frazer, S., Chameau, P., et al. (2014). Serotonin receptor $3 \mathrm{~A}$ controls interneuron migration into the neocortex. Nat. Commun. 5:5524. doi: 10.1038/ncomms6524

Nguyen, L., Rigo, J. M., Rocher, V., Belachew, S., Malgrange, B., Rogister, B., et al. (2001). Neurotransmitters as early signals for central nervous system development. Cell Tissue Res. 305, 187-202. doi: 10.1007/s004410000343

Nowakowski, T. J., Bhaduri, A., Pollen, A. A., Alvarado, B., Mostajo-Radji, M. A., Di Lullo, E., et al. (2017). Spatiotemporal gene expression trajectories reveal developmental hierarchies of the human cortex. Science 358, 1318-1323. doi: $10.1126 /$ science.aap 8809

Ojeda, J., and Avila, A. (2019). Early actions of neurotransmitters during cortex development and maturation of reprogrammed neurons. Front. Synaptic Neurosci. 11:33. doi: 10.3389/fnsyn.2019.00033

Owens, D. F., and Kriegstein, A. R. (2002). Is there more to GABA than synaptic inhibition? Nat. Rev. Neurosci. 3, 715-727. doi: 10.1038/nrn919

Pinson, A., Namba, T., and Huttner, W. B. (2019). Malformations of human neocortex in development - their progenitor cell basis and experimental model systems. Front. Cell Neurosci. 13:305. doi: 10.3389/fncel.2019. 00305

Pollen, A. A., Nowakowski, T. J., Shuga, J., Wang, X., Leyrat, A. A., Lui, J. H., et al. (2014). Low-coverage single-cell mRNA sequencing reveals cellular heterogeneity and activated signaling pathways in developing cerebral cortex. Nat. Biotechnol. 32, 1053-1058. doi: 10.1038/nbt.2967

Rakic, P. (2009). Evolution of the neocortex: a perspective from developmental biology. Nat. Rev. Neurosci. 10, 724-735. doi: 10.1038/nrn2719 
Reillo, I., De Juan Romero, C., Garcia-Cabezas, M. A., and Borrell, V. (2011). A role for intermediate radial glia in the tangential expansion of the mammalian cerebral cortex. Cereb. Cortex 21, 1674-1694. doi: 10.1093/cercor/ bhq238

Represa, A., and Ben-Ari, Y. (2005). Trophic actions of GABA on neuronal development. Trends Neurosci. 28, 278-283. doi: 10.1016/j.tins.2005.03.010

Riedel, G., Platt, B., and Micheau, J. (2003). Glutamate receptor function in learning and memory. Behav. Brain Res. 140, 1-47. doi: 10.1016/j.jad.2015. 11.021

Rojas, D. C., Singel, D., Steinmetz, S., Hepburn, S., and Brown, M. S. (2014). Decreased left perisylvian GABA concentration in children with autism and unaffected siblings. Neuroimage 86, 28-34. doi: 10.1016/j.neuroimage.2013.01. 045

Salazar, P., Velasco-Velazquez, M. A., and Velasco, I. (2008). GABA effects during neuronal differentiation of stem cells. Neurochem. Res. 33, 1546-1557. doi: 10.1007/s11064-008-9642-8

Shenoda, B. B. (2017). An overview of the mechanisms of abnormal GABAergic interneuronal cortical migration associated with prenatal ethanol exposure. Neurochem. Res. 42, 1279-1287. doi: 10.1007/s11064-016-2169-5

Suzuki, M., Nelson, A. D., Eickstaedt, J. B., Wallace, K., Wright, L. S., and Svendsen, C. N. (2006). Glutamate enhances proliferation and neurogenesis in human neural progenitor cell cultures derived from the fetal cortex. Eur. J. Neurosci. 24, 645-653. doi: 10.1111/j.1460-9568.2006.04957.x

Tanaka, D. H., and Nakajima, K. (2012). Migratory pathways of GABAergic interneurons when they enter the neocortex. Eur. J. Neurosci. 35, 1655-1660. doi: 10.1111/j.1460-9568.2012.08111.x

Vitali, I., Fievre, S., Telley, L., Oberst, P., Bariselli, S., Frangeul, L., et al. (2018). Progenitor hyperpolarization regulates the sequential generation of neuronal subtypes in the developing neocortex. Cell 174, 1264-1276. doi: 10.1016/j.cell. 2018.06.036

Wilsch-Bräuninger, M., Florio, M., and Huttner, W. B. (2016). Neocortex expansion in development and evolution - from cell biology to single genes. Curr. Opin. Neurobiol. 39, 122-132. doi: 10.1016/j.conb.2016. 05.004

Yang, C. J., Tan, H. P., and Du, Y. J. (2014). The developmental disruptions of serotonin signaling may involved in autism during early brain development. Neuroscience 267, 1-10. doi: 10.1016/j.neuroscience.2014.02.021
Yoneyama, M., Nakamichi, N., Fukui, M., Kitayama, T., Georgiev, D. D., Makanga, J. O., et al. (2008). Promotion of neuronal differentiation through activation of N-methyl-D-aspartate receptors transiently expressed by undifferentiated neural progenitor cells in fetal rat neocortex. J Neurosci. Res. 86, 2392-2402. doi: 10.1002/jnr.21696

Yuzwa, S. A., Yang, G., Borrett, M. J., Clarke, G., Cancino, G. I., Zahr, S. K., et al. (2016). Proneurogenic ligands defined by modeling developing cortex growth factor communication networks. Neuron 91, 988-1004. doi: 10.1016/j.neuron. 2016.07.037

Zhang, Y., and Bhavnani, B. R. (2006). Glutamate-induced apoptosis in neuronal cells is mediated via caspase-dependent and independent mechanisms involving calpain and caspase-3 proteases as well as apoptosis inducing factor (AIF) and this process is inhibited by equine estrogens. BMC Neurosci. 7:49. doi: 10.1186/1471-2202-7-49

Zhao, L., Jiao, Q., Chen, X., Yang, P., Zhao, B., Zheng, P., et al. (2012). mGluR5 is involved in proliferation of rat neural progenitor cells exposed to hypoxia with activation of mitogen-activated protein kinase signaling pathway. J. Neurosci. Res. 90, 447-460. doi: 10.1002/jnr.22751

Zhao, L., Jiao, Q., Yang, P., Chen, X., Zhang, J., Zhao, B., et al. (2011). Metabotropic glutamate receptor 5 promotes proliferation of human neural stem/progenitor cells with activation of mitogen-activated protein kinases signaling pathway in vitro. Neuroscience 192, 185-194. doi: 10.1016/j.neuroscience.2011. 06.044

Zheng, Z., Zhu, T., Qu, Y., and Mu, D. (2016). Blood glutamate levels in autism spectrum disorder: a systematic review and meta-analysis. PLoS ONE 11:e0158688. doi: 10.1371/journal.pone.0158688

Conflict of Interest: The authors declare that the research was conducted in the absence of any commercial or financial relationships that could be construed as a potential conflict of interest.

Copyright (c) 2020 Xing and Huttner. This is an open-access article distributed under the terms of the Creative Commons Attribution License (CC BY). The use, distribution or reproduction in other forums is permitted, provided the original author(s) and the copyright owner(s) are credited and that the original publication in this journal is cited, in accordance with accepted academic practice. No use, distribution or reproduction is permitted which does not comply with these terms. 